\title{
Mobile Station Movement Direction Prediction (MMDP) Based Handover Scanning for Mobile WiMAX System.
}

\begin{abstract}
Mobile WiMAX is a broadband technology that is capable of delivering triple play services (voice, data, and video). However, mobility in mobile WiMAX system is still an issue when the mobile station (MS) moves and its connection is handed over between base stations (BSs). In the handover process, scanning is one of the required phases to find the target BS. During the handover scanning process, the MS must synchronize with all the advertised neighbour BSs (nBSs) to select the best BS candidate for the incoming handover action. Without terminating the connection between the SBS and MS, the SBS will schedule the scanning intervals and sleep-intervals (also called interleaving interval) to MS for the handover scanning. However, during the scanning interval period, all the coming transmissions will be paused. Therefore, the redundant or unnecessary scanning of neighbouring BS cause delay and MAC overhead which may affect real-time applications. In this paper, the MS movement direction prediction (MMDP) based handover scanning scheme is introduced to overcome the mobile WiMAX handover scanning issue. It based on dividing the BS coverage area is into zones and sectors. According to the signal quality; there are three zones, no handover (NoHO), low handover (Low-HO) and high handover (High-HO) zones respectively and six sectors. In this scheme, only two BSs can become candidates; the two that the MS moves toward them will be chosen as the candidate for the handover scanning purpose. Hence, the handover scanning process repetition will be reduced with these two shortlisted BS candidates instead of scanning all nBSs. Thus, MMDP will reduce scanning delay and the number of exchange messages during the handover scanning comparing to the conventional scanning scheme. Although, the MMDP may need an extra computational time, the prediction and scanning process will be finished before the MS reach the High-HO zone, which mean the end-user's running application will be affected. Simulation results show that the proposed MMDP scheme reduces the total handover scanning delay and scanning interval duration by 25 and $50 \%$ respectively. Also, the size of scanning message is reduced, which leads to reduced signalling overhead.
\end{abstract}

Keyword: Handover; Scanning; Mobile WiMAX; Movement prediction 